\title{
Miranda
}

Revue pluridisciplinaire du monde anglophone /

Multidisciplinary peer-reviewed journal on the English-

speaking world

$17 \mid 2018$

Paysages et héritages de David Bowie

\section{Christophe Gelly, Gilles Menegaldo, Lovecraft au prisme de l'image}

Isabelle Schmitt-Pitiot

URL : http://journals.openedition.org/miranda/14133

DOI : 10.4000/miranda.14133

ISSN : 2108-6559

Éditeur

Université Toulouse - Jean Jaurès

\section{Référence électronique}

Isabelle Schmitt-Pitiot, "Christophe Gelly, Gilles Menegaldo, Lovecraft au prisme de l'image », Miranda

[En ligne], 17 | 2018, mis en ligne le 01 octobre 2018, consulté le 16 février 2021. URL : http:// journals.openedition.org/miranda/14133; DOI : https://doi.org/10.4000/miranda.14133

Ce document a été généré automatiquement le 16 février 2021.

\section{cc) (i) () $\Theta$}

Miranda is licensed under a Creative Commons Attribution-NonCommercial-NoDerivatives 4.0 International License. 


\title{
Christophe Gelly, Gilles Menegaldo, Lovecraft au prisme de l'image
}

\author{
Isabelle Schmitt-Pitiot
}

\section{RÉFÉRENCE}

Christophe Gelly, Gilles Menegaldo, Lovecraft au prisme de l'image (Cadillon : Le Visage Vert, 2017), 356 p, ISBN : 978- 2-918061-77-9

1 Face à l'extraordinaire présence dans la culture populaire contemporaine de Howard Phillips Lovecraft (1890-1937), on se plaît à rêver de sujets de dissertation à l'ancienne, demandant d'imaginer la conversation entre « le reclus de Providence » et les membres du groupe Metallica ou un auteur de bandes dessinées comme Philippe Druillet ou les concepteurs du jeu vidéo Call of Cthulhu, pour ne citer que quelques-unes des multiples références du présent ouvrage. À n'en pas douter, les seize études qu'il réunit autour de la notion d'illustration fourniraient en abondance de quoi nourrir ces conversations imaginaires.

2 Cette richesse s'offre facilement au lecteur, en dépit de la réputation d'hermétisme de Lovecraft, car contrairement à beaucoup d'ouvrages constitués d'articles d'auteurs différents, ce recueil se caractérise par la qualité de sa structure. En effet, les articles sont répartis en quatre chapitres: Lovecraft et l'image, Lovecraft et le cinéma, Lovecraft et la bande dessinée et enfin Lovecraft figure transmédiale. Ils permettent un vaste tour d'horizon de la relation des écrits de Lovecraft aux images de tout ordre, et plus largement aux différents médiums de création et de communication qui continuent de s'approprier cette œuvre, source de repères culturels contemporains majeurs. Le prisme du titre symbolise bien la démarche d'ensemble, la question de la représentation étant envisagée à chaque fois selon un angle différent et chaque auteur jetant un éclairage nouveau et intriguant sur une œuvre qui revient sans cesse sur le thème même de l'irreprésentabilité. 
3 Premier paradoxe et première évidence pourtant, l'iconographie est abondante et indispensable pour illustrer la tension entre écrit et image. Soulignons son exceptionnelle qualité, l'illustration de Philippe Jozelon en tête de l'introduction constituant pour nous la plus belle entrée en matière puisque cette image blanche et bleu nuit de marches montant vers l'invisible donne à voir au seuil du livre à la fois le désir et l'effroi des lecteurs et lectrices de Lovecraft.

4 L'introduction joue parfaitement son rôle : brève, elle pose le cadre général et inscrit le recueil dans une réflexion sur l'adaptation, au sens large tel que Linda Hutcheon le définit, ou plutôt sur les hypertextes visuels, graphiques, filmiques... d'une œuvre liée à la notion d'illustration dès son écriture, Lovecraft laissant lui-même des blancs comme autant d'invites. Au-delà, l'ouvrage, qui a pour projet de parcourir le champ des rapports entre cette œuvre et ses continuateurs, va creuser la question de la représentation visuelle d'un corpus qui n'a de cesse d'insister sur la difficulté de la représentation du monstrueux. En d'autres termes, comment illustrer des textes qui insistent sur l'indicible?

5 On entre dans le premier chapitre, consacré aux relations texte/image dans l'œuvre même de Lovecraft, par la grâce d'un texte de Denis Mellier que l'on peut placer en exergue $\mathrm{du}$ volume entier. Il y montre qu'en dépit des qualificatifs privatifs, innommable, indicible, irreprésentable, qui saturent le corpus autant que le discours des exégètes, l'écriture de Lovecraft, éminemment descriptive, visant « l'hyperréalisme des irréalités ", devient le lieu même de la transformation de la lettre en image. Là se joue la tension extrême entre indétermination et présence, crise de la figuration qui obsède à leur tour les nombreux auteurs de bandes dessinées que Denis Mellier étudie.

6 Traitant de l'intertextualité dans At the Mountains of Madness (1931), Lauric Guillaud ajoute à Poe et Verne le journal de Lewis, dont la quête, associant l'horreur et l'émerveillement, résonne de l'appel du gouffre et débouche fatalement sur le réveil du monstre, la folie et/ou la mort. Au-delà de ces sources littéraires, Eric Lysøe analyse comment, dans le même roman, Lovecraft convoque explicitement la peinture de Nicolas Roerich, qui déplace et dépasse les perspectives au-delà du vertige pour définir ce nouveau fantastique, " art cosmique non surnaturel ", vers lequel tend Lovecraft. Roger Bozetto poursuit ce travail sur l'art lovecraftien de simuler un réel inconcevable par le biais du ressassement, de l'oxymore et de la prétérition, qui permettent à des espaces contradictoires de coexister, tandis que Julien Schuh affine l'analyse des images chez Lovecraft en s'appuyant sur trois exemples, un dessin occulte, un graphique scientifique et une œuvre d'art futuriste, pour parvenir à la conclusion que ces images remplacent une présence impossible.

7 Lovecraft professait un certain mépris pour le cinéma, comme le rappellent les quatre articles du chapitre explorant la problématique de l'adaptation de son œuvre à l'écran et Philippe Met demande avec quelque malice si ce n'est pas la raison pour laquelle ces adaptations sont assez tardives et pour la plupart peu convaincantes. Néanmoins, cette fortune fluctuante est davantage à rattacher à la question même de l'adaptation filmique, comme le montrent les ouvrages ayant du mal à cerner et classifier un corpus d'adaptations de Lovecraft, la frontière étant mouvante entre les « vraies » adaptations et les films inspirés par les thèmes et tropes lovecraftiens. Les articles vont donc s'attacher à définir la nature et les formes cinématographiques du matériau source, dont certaines caractéristiques, comme la passivité des protagonistes ou la rareté des personnages féminins, seraient pour certains critiques des entraves à la représentation 
filmique. Cependant, Philippe Met présente en détail la manière qu'a Lucio Fulci de s'approprier les motifs propres à Lovecraft au début des années 1980, tandis que Christophe Chambost compare The Haunted Palace de Roger Corman (1963) et The Resurrected de Dan O'Bannon (1991). Il montre comment le premier utilise la référence à Poe pour approcher l'horreur gothique et privilégier l'élément humain alors que le second a davantage recours à l'horreur grotesque, tâchant de reproduire l'effet de sidération que recherchait Lovecraft. Gilles Menegaldo revient sur The Haunted Palace et ses effets visuels de défamiliarisation avant de présenter plusieurs films de John Carpenter, en particulier Prince of Darkness (1987) et surtout In the Mouth of Madness (1995), mettant en scène une confrontation de la lettre et de l'image au moyen d'un réseau intertextuel très dense. Pierre Jailloux revient lui aussi sur la question de la monstration ou mise en scène du monstrueux sous l'angle du found footage, soit un document filmique informatif, un fantastique objectif se concentrant sur l'énonciation plutôt que sur un énoncé irreprésentable. Dans la lignée de Paranormal Activity (Oren Peli, 2009), l'être humain s'y retrouve filmé par le monstre et c'est le médium lui-même qui manifeste la sidération et l'effroi.

$8 \quad$ La bande dessinée n'a de cesse elle aussi de mettre en images cet irreprésentable, et ses auteurs de travailler la pâte lovecraftienne chacun à sa manière. Christophe Gelly montre ainsi comment Alan Moore (scénario) et Jacen Burrow (dessin) adaptent l'univers de Lovecraft dans leur album Neonomicon, qui se présente comme une nouvelle version du Necronomicon, source imaginaire de Lovecraft que Moore pourvoit d'une ligne narrative, mettant à distance le mythe lovecraftien par le biais d'une parodie introduisant les thèmes du racisme et de la répression sexuelle. Moore et Burrows représentent un monstre sexualisé et composite qui suggère une intimité entre lui et le monde humain qu'il regarde et bouleverse. Pour Jérôme Dutel, les différentes adaptations en bandes dessinées de la nouvelle "The Outsider » (1921) parviennent à illustrer plus ou moins bien l'obsession de Lovecraft pour cette confrontation entre l'homme et l'Autre. Sa comparaison du travail d'Horacio Lalia, d'Hernan Rodriguez, d'Erik Kriek et de Gou Tanabe l'amène à considérer l'œuvre de ce dernier comme la plus accomplie, c'est-à-dire celle qui, d'après lui, parvient le mieux à recréer l'étrangeté de sa source. De son côté, Karen Vergnol-Rémont rend un bel hommage au dessinateur argentin Alberto Breccia en analysant de très près son adaptation de «The Haunter of the Dark »/«Celui qui hantait les ténèbres » dans un album faisant le choix du noir et blanc et du mélange des techniques, des formes et de l'informe pour laisser la place au seul texte de dans l'ultime vignette.

9 Les mythes, les créatures et les atmosphères engendrés par un auteur esseulé et méconnu de son vivant se voient désormais prolongés, voire augmentés, dans des médiums nouveaux comme les jeux de rôles. Isabelle Périer étudie ces nouvelles formes d'adaptation se présentant comme des ouvrages composites. Ainsi, ceux dédiés à la cité de Kadath, inventée par Lovecraft, associent illustrations, textes, nouvelles traductions, guide touristique imaginaire et règles du jeu comme autant de satellites du texte source, au point que l'on peut se demander si les limites de l'adaptation définies par Linda Hutcheon ne sont pas dépassées et s'il ne conviendrait pas de suivre Richard Saint-Gelais dans ses Fictions transfuges et de parler plutôt d'une communauté thématique, structurelle et poétique entre des objets aussi différents. Les raisons de cette extraordinaire pérennité de Lovecraft dans la culture populaire contemporaine sont développées ensuite par Rémi Cayatte, et tiennent pour lui à la présence récurrente de thèmes et de motifs (les monstres, l'enquête, la folie et enfin la 
dangerosité de la quête de connaissance) universels. Enfin, deux adaptations particulières sont présentées, l'une et l'autre sous l'angle du paradoxe, ultimes preuves de l'infinie labilité des créations lovecraftiennes. Pour Arnaud Moussard, Night Gaunts (1988-1990) de Brett Rutherford adapte un auteur qui n'a jamais écrit pour le théâtre même s'il avait beaucoup apprécié The King in Yellow de Robert William Chambers (1895). Le paradoxe se renforce dans la mesure où l'argument biographique donne lieu à un "diptyque féminin " autour de la mère, puis de l'épouse de Lovecraft. Autre facette du prisme, Christopher L. Robinson cherche dans les tableaux de H.R. Giger des preuves de l'inspiration lovecraftienne revendiquée par ce dernier dans ses deux recueils de peintures intitulés Necronomicon, alors que l'on peine à y retrouver l'univers du mythe de Cthulhu. Cependant un jeu d'associations mystérieuses, d'allusions, d'échos permet de relier Giger à Lovecraft.

À la suite de l'écrivain, luttant pour écrire l'indicible en associant le flou et le précis dans une formule toujours instable, ses multiples et divers illustrateurs et adaptateurs remettent à chaque fois sur le métier la question de la représentation, et les éditeurs du recueil ont eu l'excellente idée de laisser le dernier mot, non pas à un universitaire, mais à un dessinateur ayant l'expérience de l'illustration de Lovecraft. Pour lui, cela consiste à mettre en scène l'effroi de piètres protagonistes face à l'abîme. Illustrer/ adapter Lovecraft, ou figurer ses/nos cauchemars.

\section{INDEX}

Mots-clés : Lovecraft, illustration, bandes dessinées, cinéma, adaptation, intermédialité

Keywords : Lovecraft, illustration, comics, cinema, adaptation, inter-media

\section{AUTEURS}

\section{ISABELLE SCHMITT-PITIOT}

Maître de Conférences

Université de Bourgogne-Franche-Comté

isabelle.schmitt@u-bourgogne.fr 